\title{
ANÁLISIS DE UN JUGO DE MORA (Rubus adenotrichos) ENDULZADO CON ESTEVIA (Stevia rebaydina Bertoni), UNA APROXIMACIÓN METABOLÓMICA
}

\section{ANALYSIS OF JUICE BLACKBERRY (Rubus adenotrichos) SWEETENED WITH STEVIA (Stevia rebaudina Bertoni), A METABOLOMICS APPROACH}

\author{
César González Torrivilla ${ }^{1}$, Lorena Hernández², Fabrice Vaillant Barka ${ }^{3}$
}

\begin{abstract}
${ }^{1}$ Doctor en Ciencias, mención Ciencia y Tecnología de Alimentos, Departamento de Procesos Agroindustriales, Decanato de Agronomía. Universidad Centroccidental "Lisandro Alvarado" (UCLA), Av. Florencio Jiménez, Núcleo Obelisco. Barquisimeto - Lara, Venezuela, Código Postal 3001. Teléfono: 0058 4165539861, Dirección de correspondencia, email: cesargonzalez@ ucla.edu.ve; ${ }^{2}$ Lic. en Química, Especialista en Espectrometría de Masas, Centro de Investigación en Productos Naturales, Ciudad de la investigación. Universidad de Costa Rica, Código Postal 11501-2060 San José, Costa Rica, email: lorena.hernandez@ucr.ac.cr; ${ }^{3}$ Doctor en Ingeniería de Procesos Agroalimentarios, Centre de Coopération Internationale en Recherche Agronomique pour le Développement (CIRAD), UMR 95 QUIALISUD, Montpellier, France, e-mail: fabrice.vaillant@cirad.fr
\end{abstract}

Rev. U.D.C.A Act. \& Div. Cient. 20(1): 121-129, Enero Junio, 2017

\section{RESUMEN}

La investigación tuvo como propósito analizar, a través de una aproximación de estudio metabolómico, la composición integral de un jugo microfiltrado a base de mora (Rubus adenotrichos), endulzado con hojas deshidratadas de estevia (Stevia rebaudiana Bertoni) y su comparación con análogos obtenidos por tratamiento térmico convencional, endulzados con sacarosa. Para el registro de la información espectral, se optó por un enfoque no dirigido, en el que se midieron, simultáneamente, tantos metabolitos, como fue posible, en los cuatro tipos de muestras elaboradas (jugo microfiltrado no pasteurizado y pasteurizado, endulzado con estevia y sacarosa). Fue empleado un sistema de Cromatografia Líquida de Ultra Alta Resolución (UPLC), equipado con un detector de red de fotodiodos (PDA) acoplado a un electrospray de ionización y dispuesto con Q-TOF al espectrómetro de masas (ESI-Q-TOF/MS). Con base en los archivos formato SPL, aportados por el UPLC y, una vez convertidos a formato NetCDF, se efectuó el análisis respectivo, en el software MZmine, el cual, detectó cada uno de los iones presentes, discriminando la presencia o no de los mismos, en las diferentes muestras, identificándolos según su masa (molecular) y el tiempo de retención donde fueron registrados en el espectro, por el espectrómetro de masas. De acuerdo con las deducciones generadas, el análisis estadístico aplicado, confirmó la variación de la composición del jugo endulzado, tanto con estevia como con sacarosa, al ser sometidos a tratamiento térmico, al corroborar diferencias estadísticamente significativas, lo que permite afirmar que el tratamiento, para las formulaciones estudiadas, genera una variación de los iones presentes.

Palabras clave: Iones, metabolitos, microfiltración, pasteurización.

\section{SUMMARY}

The purpose of the research was to analyze, through a metabolomic study, the overall composition of a microfiltration blackberry juice (Rubus adenotrichous) sweetened with dried leaves of stevia (Stevia rebaudiana Bertoni) and its comparison with analogues obtained by conventional heat treatment, sweetened with sucrose. The spectral information was obtained by an undirected approach. all possible metabolites produced in the four samples (unpasteurized and pasteurized microfiltered juice, sweetened with stevia and sucrose) were measured simultaneously. Analyses were conducted in series using a Waters Acquity Ultra Performance Liquid Chromatography system (UPLC) equipped with a photodiode array detector (PDA) and coupled with Electrospray Ionization and Quadrupole Time-Of-Flight Mass Spectrometry (ESI-Q-TOF/MS. SPL format files, provided by the UPLC, in the analysis of the samples were converted to NetCDF format, the respective analyzes were performed by the MZmine software. all ions were detected, discriminating presence or not in the different samples. The mass (molecular) and the retention time were recorded by the mass spectrometer. The results of the statistical analysis applied confirmed the variation in composition microfiltration 
blackberry juice sweetened with sucrose and stevia when subjected to heat treatment step, corroborating statistically significant differences between them, which allows to affirm that the treatment for the formulations studied generates a variation of the ions present.

Key words: Ions, metabolites, microfiltration, pasteurization (CAB Thesaurus).

\section{INTRODUCCIÓN}

En la actualidad, la demanda mundial por edulcorantes naturales ha sido apreciable, debido a los diversos efectos secundarios asociados al consumo de edulcorantes sintéticos (Keerthi et al. 2011). Un número reducido de compuestos edulcorantes, de este tipo, bajo en calorías y disponibles en la naturaleza, se utilizan comercialmente en diversos productos, como, por ejemplo: la taumatina, la glicirricina, el xilitol, el phyllodulcin, el mogrósido y el esteviósido (Puri et al. 2011). Entre ellos, se destaca el interés que, en la última década, han generado los edulcorantes, obtenidos a partir de las hojas de la planta Stevia rebaudiana Bertoni, los cuales, se encuentran disponibles en el comercio, en diversos países, donde se han utilizado durante varias décadas para endulzar una gran variedad de alimentos, incluyendo, bebidas, verduras dulces, encurtidos y mariscos (Sharoba et al. 2012).

En este orden de ideas, y dada la importancia que el consumidor moderno le otorga a aquellos componentes de los alimentos que, a pesar de no ser nutricionalmente esenciales, puedan promover su bienestar y ayudarle a reducir el riesgo de presentar enfermedades crónicas y degenerativas; el uso de los esteviolglucósidos, como edulcorante y saborizante en la formulación de alimentos, resulta prometedora (González et al. 2014).

A este respecto, el aumento sostenido del consumo de zumos procesados, evidenciado por la incorporación de una amplia gama de productos pertenecientes a esta categoría, favorecen su empleo para la fabricación de bebidas, concretamente, para la producción de jugos naturales. A pesar de los avances tecnológicos, las industrias procesadoras de jugos de frutas aplican, principalmente, operaciones unitarias convencionales para la extracción, la filtración y la concentración de pulpas, a partir de los frutos enteros. La elección del equipamiento más adecuado para lograr cada uno de estos propósitos, está determinado por muchos factores, que deben ser sopesados cuidadosamente, para asegurarse del cumplimiento de los requisitos del proceso y el costo de capital. Estos factores, incluyen, entre otras consideraciones, las propiedades del material de alimentación, los requisitos de calidad del producto, las condiciones de funcionamiento y el costo de operación (Prada et al. 2004). Bajo las condi- ciones actuales de producción industrial de zumos de frutas es inevitable que sufran alteraciones en su metabolismo, por las propiedades y la intensidad de los tratamientos aplicados. Dada la poca identificación de afectaciones concretas, que caractericen el nivel de impacto de estos procesos en el estatus metabólico y su vinculación con la calidad final de los productos, el abordaje de este impacto, implica el uso combinado de diferentes metodologías y el análisis de algunos grupos específicos de sustancias trazadores de cambios, en el metabolismo de estos productos.

Actualmente, se presenta gran interés en las técnicas ómicas, como la metabolómica, referida al conjunto de ciencias y de técnicas dedicadas al estudio completo del sistema constituido por el conjunto de moléculas que constituyen los intermediarios metabólicos, metabolitos, hormonas y otras moléculas, y los metabolitos secundarios, que se pueden encontrar en un sistema biológico (Oliver et al. 1998). El estudio de la metabolómica de los alimentos ha aumentado gradualmente en los últimos años, porque los sistemas alimentarios están directamente relacionados con la nutrición y la salud humana (Kim et al. 2016); sin embargo, el conjunto completo de los metabolitos de pequeñas moléculas presentes en los alimentos que componen la dieta humana y el papel de los sistemas de producción de alimentos en la alteración de este metaboloma de los alimentos son todavía ampliamente desconocidos (Johanningsmeier at al. 2016).

Esta técnica ha sido aplicada en la evaluación de frutas y derivados, principalmente, en aspectos relacionados con la identificación de variedades, diferenciación de la calidad y propiedades organolépticas (nuevos marcadores químicos de calidad sensorial), determinación de autenticidad y adulteraciones en zumos y derivados, evaluación nutrabólica y desarrollo de alimentos funcionales, entre otros. Respecto a su uso, en estudios de evaluación de tecnologías de conservación de alimentos, es un campo de aplicación explorado a baja escala por la comunidad científica, que no proyecta, a la fecha, una producción científica destacable (Vaclavik et al. 2012; Cajka et al. 2011).

Sobre la base de las ideas expuestas, la presente investigación apuntó esfuerzos en la evaluación de un jugo de mora microfiltrado, dirigido a consumidores con regímenes especiales de alimentación, a fin de evidenciar, por medio de un estudio metabolómico preliminar, diferencias entre jugos endulzados, tanto con sacarosa como con estevia y análogos sometidos a una etapa de pasteurización, como operación de conservación.

\section{MATERIALES Y MÉTODOS}

Todas las actividades que comprenden la formulación y la elaboración del jugo de mora fueron realizadas en las ins- 
talaciones del Centro Nacional de Ciencia y Tecnología de Alimentos (CITA), Universidad de Costa Rica. Se emplearon moras (Rubus adenotrichos) de la variedad "vino con espinas", en estado de madurez fisiológico y congeladas a $-20^{\circ} \mathrm{C}$. Para la obtención de la pulpa refinada, se sometió la totalidad del lote de moras, a una etapa de prensado. La pulpa refinada obtenida fue envasada en tambores plásticos desinfectados y almacenada bajo condiciones de congelación $\left(-20^{\circ} \mathrm{C}\right)$. El jugo formulado fue endulzado con hojas de Stevia rebaudiana deshidratadas en polvo, de la variedad Morita II, producida por un productor del municipio Sucre, Aragua, Venezuela. La determinación de su proporción en la formulación de uno de los jugos formulados, se rigió por criterios sensoriales, a través de dos pruebas afectivas, celebradas con anterioridad, en las cuales, se definió $2,5 \mathrm{~g} /$ Ljugo. Una vez formulados los jugos endulzados, tanto con sacarosa como con estevia, fueron sometidos a una etapa de microfiltración tangencial. El sistema de microfiltración estuvo conformado por una bomba centrífuga, para el suministro del flujo tangencial; una bomba de desplazamiento positivo, que condujo la alimentación y un intercambiador de calor tubular, para el control de la temperatura. Se mantuvo la temperatura del jugo a $35^{\circ} \mathrm{C}$, para evitar la disminución de la aceptación sensorial del jugo en estudio. La membrana empleada durante la etapa de microfiltración posee un tamaño de poro igual a $10 \mathrm{~nm}$, de $5 \mathrm{kDa}$, área de filtración de $0,33 \mathrm{~m}^{2}$, fabricada por Pall Corporation, USA. Los ensayos de microfiltración, se iniciaron en condiciones de presión, a través de la membrana igual a 2,54bar y un flujo de permeado de $270 \mathrm{~L} / \mathrm{hm}^{2}$. Dichos ensayos, se efectuaron a una temperatura constante, con una presión transmembranaria constante, una velocidad tangencial constante y un tiempo aproximado de 70 min.

El permeado obtenido fue envasado asépticamente, mediante un sistema acoplado a la salida del sistema de MF, conformado por una cámara hermética, con inyección constante de nitrógeno, con instalación interna de una lámpara de luz ultravioleta. El jugo fue envasado en bolsas especiales, a base de un laminado, compuesto por capas interpuestas de polietileno-aluminio-polietileno. La mitad de las bolsas de cada formulación fue sometida a una pasteurización, introduciéndolas en una marmita de doble chaqueta (calentada con vapor), dispuesta con agua potable. Se estableció, con base en pruebas preliminares (carga inicial de 7,5 x 104UFC/ $\mathrm{mL}$, determinada mediante la realización de pruebas microbiológicas) y en valores referenciales obtenidos de la literatura (valor $\mathrm{Z}=5,5^{\circ} \mathrm{C}$ y tiempo de reducción decimal $\mathrm{D}=5$ minutos), como condiciones de pasteurización: $90^{\circ} \mathrm{C}$ por 30 segundos. Transcurrido el tiempo, eran removidas y sumergidas en un baño de agua helada. Cada una de las cuatro muestras (jugo MF endulzado con estevia sin pasteurizar, jugo MF endulzado con estevia pasteurizado, jugo MF endulzado sacarosa sin pasteurizar y jugo MF endulzado sacarosa pasteurizado) fueron almacenadas durante un mes, bajo 5 condiciones de temperatura distintas: $5^{\circ} \mathrm{C}, 20^{\circ} \mathrm{C}, 30^{\circ} \mathrm{C}, 37^{\circ} \mathrm{C}$ y $45^{\circ} \mathrm{C}$, en cavas especiales, acondicionadas para tal fin.

Con el propósito de analizar el conjunto de iones presentes y así evidenciar similitudes o diferencias entre su información espectral, se optó por un enfoque no dirigido, en el que se midieron, simultáneamente, tantos iones, como fue posible. Se tomaron algunas de las consideraciones, señaladas en estudios previos, principalmente, los efectuados por Keerthi et al. (2011), Rodríguez, et al. (2013) y García et al. (2014). Su ejecución fue realizada en las instalaciones del Centro de Investigaciones de Productos Naturales (CIPRONA) de la Universidad de Costa Rica. A continuación, se describen cada una de las actividades involucradas para el análisis de las muestras:

Preparación de muestras para análisis metabolómico: Se extrajo, dos veces por semana, una porción de cada muestra almacenada, a las cinco condiciones diferentes; el procedimiento, se realizó en el interior de una campana de flujo laminar. Las muestras fueron preservadas en tubos eppendorf de $1,5 \mathrm{~mL}$ y conservadas a $-70^{\circ} \mathrm{C}$. El día estipulado para el análisis, todas las muestras fueron descongeladas, centrifugadas (14000rpm por 10 minutos), diluidas con agua grado HPLC en proporción 1:1 y trasvasadas a los viales para introducirlas al UPLC.

Sistema CL-MS empleado: Los análisis fueron realizados en un sistema de Cromatografia Líquida de Ultra Alta Resolución (UPLC) de Waters Acquity, el cual, estuvo equipado con un detector de red de fotodiodos (PDA), acoplado a un electro spray de ionización (tensión capilar de 2,3 kV, temperatura seca $270^{\circ} \mathrm{C}$ ) y dispuesto con Q-TOF (analizador cuádruplo de tiempo de vuelo) al espectrómetro de masas (ESI-Q-TOF/MS) (Waters SYNAPT G1, Waters Corporation, Milford, MA, EE.UU.). El equipo estaba dotado, además, de un dosificador binario de disolvente, un muestreador automático y un desgasificador, controlado por MassLynx V4.1 (Waters Corporation, MA, EE.UU.); las condiciones cromatográficas empleadas son descritas en la tabla 1. El espectrómetro de masas fue equilibrado previamente, con las soluciones estándares correspondientes. Los experimentos de fragmentación inducida por colisión MS2, se realizaron utilizando la interfaz ESI operativa, en el modo de ion positivo, usando una velocidad de barrido de exploración de 1/s y una rampa de energía de colisión de 10 a $40 \mathrm{eV}$. La temperatura de desolvatación fue $330^{\circ} \mathrm{C}$ y el flujo de gas de desolvatación de $350 \mathrm{~L} / \mathrm{h}$. Todas las muestras fueron inyectadas por triplicado, en dos réplicas de forma aleatoria.

Análisis de los resultados obtenidos: Se convirtieron los archivos programados por el sistema acoplado de CL-MS, de formato SPL, aportados por el UPLC, a formato AFAM.raw y, 
Tabla 1. Condiciones cromatografías empleadas durante el análisis LC-MS, para el estudio metabolómico de muestras de jugo de mora, bajo los diferentes tratamientos, endulzados con esteviolglucósidos o sacarosa, sometidos o no a pasterización.

\begin{tabular}{|c|c|}
\hline VARIABLE & CONDICIONES ESPECÍFICAS EN LA MEDICIÓN \\
\hline Modo de ionización de los espectros & Modo positivo \\
\hline Rango de medición de espectros & de $50 \mathrm{~m} / \mathrm{z}$ a $1000 \mathrm{~m} / \mathrm{z}$. \\
\hline $\begin{array}{c}\text { Disolvente A } \\
\text { Disolvente B }\end{array}$ & $\mathrm{HCN}+0,01 \%$ Ácido Fórmico. \\
\hline Columna & APLCido Fórmico. \\
\hline Flujo de inyección & $0,4 \mathrm{~mL} / \mathrm{min}$. \\
\hline Volumen inyectado $18,1,0 \times 100 \mathrm{~mm}, 1,7 \mu \mathrm{m}$. \\
\hline Temperatura de la columna & $60^{\circ} \mathrm{C}$ \\
\hline La tasa de adquisición de datos & $0,02 \mathrm{~s}$ \\
\hline
\end{tabular}

por último, a NetCDF (a través del software Masslynx Databridge). Seguidamente, se efectuó el análisis respectivo, en el software especializado MZmine, el cual, detectó, organizó y enlistó, cada uno de los iones presentes, discriminando, a su vez, la presencia o no de los mismos, en las diferentes muestras, identificándolos según su masa (molecular) y el tiempo de retención, registrados en el espectro por el espectrómetro de masas. El listado detallado fue analizado estadísticamente, a través del software SIMCA 13.0.2, que permitió efectuar un análisis de componentes principales (PCA). Estableciendo diferentes comparaciones para, de esta manera, construir la data que alimentó el análisis discriminante PLS, específicamente, se empleó un análisis de tipo PLS-DA. Las comparaciones establecidas para cada análisis PLS-DA efectuado, fueron: A) Comparación de iones presentes en las muestras de jugo MF con estevia, con y sin pasteurización; B) Comparación de iones presentes en las muestras de jugo MF con sacarosa, con y sin pasteurización y, C) Comparación de iones presentes en las muestras de jugo MF con estevia y formulado con sacarosa.

El PCA registró los resultados en puntuaciones (scores) (eje de coordenadas) y plots (manchas). Las puntuaciones de las manchas en el eje de coordenadas (score-plot) permitió describir las relaciones de las muestras entre sí, visualizándose, de esta manera, las similitudes y las diferencias entre las mismas. Para ello, se empleó el software MZmize, siguiendo la metodología descrita por Pluskal et al. (2010). Con este software, por tanto, se obtuvieron dos tipos de resultados: el score plot, representado por el eje de coordenadas, donde se sitúan las muestras con base en la cantidad de iones que la conforman y los marcadores potenciales sobre o sub expresados, en las dos clases de muestras.

\section{RESULTADOS Y DISCUSIÓN}

El modelo ajustó 656 variables, de las cuales, 654 pertenecen a la variable $\mathrm{X}$ y dos son de la variable $\mathrm{Y}$; a partir de ellas, se realizaron los tres análisis PLS. A continuación, se presentan los resultados obtenidos en las tres mencionadas comparaciones establecidas.

COMPARACIÓN A: Cotejo efectuado entre muestras de jugo de mora microfiltrado formulado con estevia pasteurizado y no pasteurizado: La figura 1 presenta, en su sección A, la dispersión de las muestras en el espacio X, es decir, representa cómo se sitúan las observaciones modeladas en el espacio X; en él, se puede apreciar que las muestras pertenecientes a cada grupo (azul, para las muestras de jugo de mora microfiltrado formuladas con estevia pasteurizadas y, en color verde, las muestras de jugo de mora microfiltrado formuladas con estevia no pasteurizadas), no exhiben un patrón definido, distribuyéndose en el plano de forma indistinta.

A pesar de ello, el análisis estadístico aplicado sobre los valores de las intensidades de los diferentes iones identificados en dichas muestras y que consistió en un análisis de varianza, confirmó, estadísticamente, diferencias significativas ( $p$ valor es menor a 0,05$)$, con un nivel de confianza del $95 \%$; nótese que el valor $p$, en la prueba, fue de 0,04, ligeramente inferior al valor establecido para confirmar diferencias $(0,05)$. Al confirmar diferencias estadísticamente significativas fueron seleccionados, a través del software, los iones de mayor importancia, por su notable participación en la diferenciación de los tratamientos, en otras palabras, los iones que presentaron valores muy diferentes entre las muestras de una categoría con respecto a otra (muestras pasteurizadas y 
A)

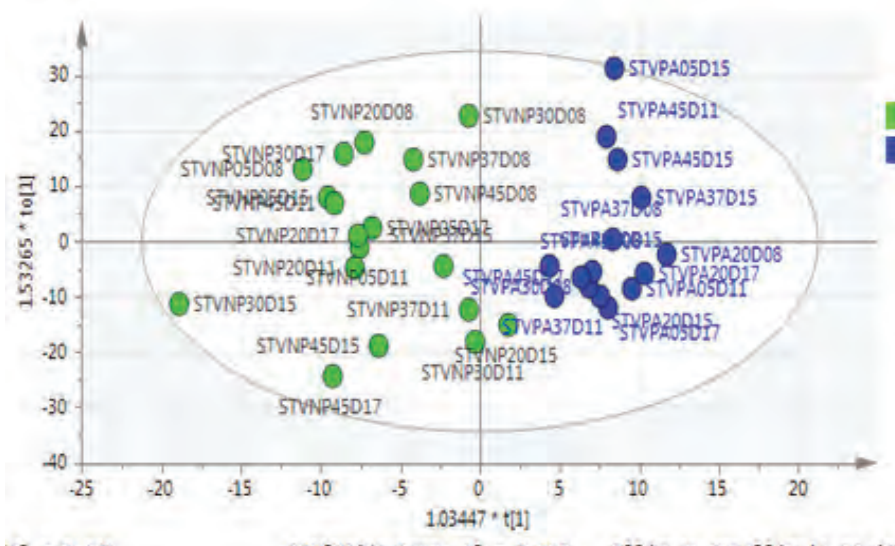

1] $=0.101$

$R .2 \times[X$ side comp. 1$]=0.265$

Ellipse: Hotelling's T2 (95\%).

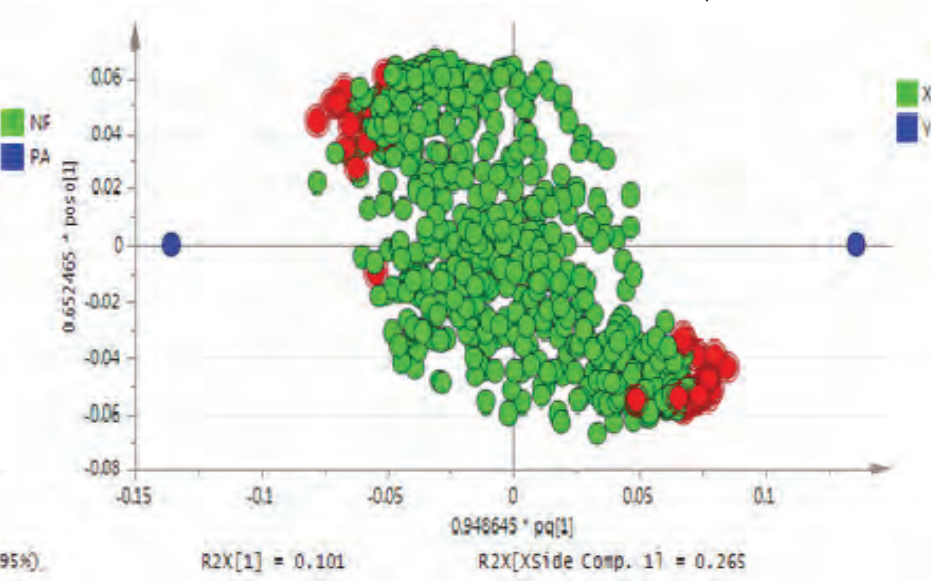

A) Representación bidimensional arrojada por el modelo OPLS-DA estimado y B) lones discriminantes (VIP) en el espacio, identificados por el software SIMCA, durante la comparación de muestras de jugo de mora microfiltrado formulado con estevia con y sin tratamiento.

Figura 1. Representación en el espacio de las muestras formuladas con estevia con y sin tratamiento.

no pasteurizadas). Ya que el valor medio de la trama VIP es 1 , esta selección consistió en señalar los iones con valores VIP más altos (por el elevado número del listado de iones arrojados por el software, se consideraron aquellos mayores o iguales a 1,5). Al seleccionar dichos iones, el software aportó el listado de dichos metabolitos, representándolos, además, en el plano, tal y como se muestra en la figura 1B.

Estos metabolitos discriminantes, por su mayor influencia en el modelo, pueden ser apreciados, en la mencionada figura, por su ubicación más lejana al origen (puntos color rojo en la figura) y, por el contrario, los iones con valores VIP, menores a este grupo, pueden ser visualizados de color verde en la figura, se ubican cerca del centro, por lo que tienen una influencia más débil el modelo.

Apoyados en los iones discriminantes arrojados por el programa como iones VIP, por ser distantes, entre las muestras tratadas o no, es decir, entre todos los 2.068 iones presentes en las muestras, este grupo presenta, en mayor medida, una intensidad distinta entre las muestras de diferente tratamiento; se construyeron los histogramas respectivos para, de una manera gráfica, poder visualizar la semejanza o no que pueda existir, según la intensidad de cada uno de los iones, entre las muestras tratadas o no (pasteurizadas o no). Dado los resultados obtenidos en las gráficas elaboradas exhiben para todos los iones empleados un comportamiento irregular, ya que no permiten definir una tendencia definida a disminuir o por el contrario aumentar entre las muestras tratadas y no tratadas, no es posible apreciar diferencias entre los tratamientos, por lo que se decidió efectuar un análisis más detallado de los datos, discriminado en cada temperatura y así evitar el error generado, producto de la comparación simultánea de todas las muestras, las cuales, fueron conservadas a diferente temperatura.

De los cinco nuevos análisis PLS-DA, correspondiente a la comparación de las muestras de jugo de mora microfiltrado endulzado con estevia a las diferentes temperaturas de almacenamiento $\left(5,20,30,37\right.$ y $\left.45^{\circ} \mathrm{C}\right)$, solo se pueden afirmar diferencias estadísticamente significativas en el grupo de muestras almacenadas a $5^{\circ} \mathrm{C}$ (en el análisis, se obtuvo un p valor menor a 0,01 , por lo que se puede avalar con un nivel de confianza del 99\%, que existen diferencias estadísticamente significativas entre las muestras formuladas con estevia pasteurizadas y no pasteurizadas, almacenadas a dicha temperatura). Siguiendo con la metodología descrita fueron seleccionados, a través del software, los iones de mayor importancia, por su participación en la diferenciación de los tratamientos. Esta selección consistió en señalar los iones con valores VIP $\geq 1,5$; el software aportó el listado de iones. Estos iones discriminantes, por su mayor influencia en el modelo, pueden ser apreciados, en la mencionada figura, por su ubicación más lejana al origen (puntos color rojo, en la figura) y, por el contrario, los iones con valores VIP, menores a este grupo, pueden ser visualizados de color verde, en la figura, se ubican cerca del centro, por lo que tienen una influencia más débil el modelo. 
Resultados comparables fueron obtenidos por Khan et al. 2017, quienes emplearon igualmente UPLC-MS, para analizar metabolitos polares y no polares de pancharishtha, una formulación tradicional poliherbal, preparada por cocción y por fermentación, de diversos vegetales. Los resultados del análisis UPLC-MS fueron empleados por los autores para efectuar un análisis multivariante, a través de XLSTAT. El diagrama de Análisis de Componentes Principales (PCA) distribuyó todas las muestras en cuatro grupos diferentes, con dos formulaciones cada uno, encontrando, de esta manera, diferencias significativas, en el contenido de las diferentes formulaciones.

Según la metodología empleada, restaría apoyarse en los iones discriminantes arrojados por el programa y, a través del software Masslynx versión 4.0, será posible la visualización de cada tiempo de retención, en el cual, fue detectado cada uno de estos iones discriminantes y los espectros de cada muestra, lo que permitiría, a su vez, la comparación de los iones asociados con bases de datos on line para, de esta manera, poder identificar con qué metabolitos pueden estar relacionados y así poder reconocer qué molécula representan.
COMPARACIÓN B: Cotejo efectuado entre muestras de jugo de mora microfiltrado formulado con sacarosa pasteurizado y no pasteurizado: El análisis efectuado por el software SIMCA, tal y como fue descrito para la comparación anterior (comparación A), permitió obtener el comportamiento de los iones presentes en las muestras, según el tratamiento. Su distribución en el espacio bidimensional puede ser observado en la figura $2 \mathrm{~A}$.

En ellas, se puede apreciar claramente, cómo las muestras pertenecientes a cada categoría se distribuyen de forma similar, agrupándose entre sí y diferenciándose por clase; nótese, cómo los puntos azules (muestras pasteurizadas) en las figuras, se ubican a la derecha y en la parte superior, con la única excepción de la muestra SANP05D01 (jugo de mora microfiltrado endulzado con sacarosa, conservado a $5^{\circ} \mathrm{C}$ y almacenado durante un día), la cual, es posición atípica, respecto al resto de las muestras de su categoría y las muestras color verde (muestras no pasteurizadas), se sitúan a la izquierda y en la parte inferior. Dicha diferenciación entre las muestras, según el tratamiento, fue corroborado por el análisis de varianza efectuado, al alcanzar un $\mathrm{p}$ valor inferior a 0,05 , lo que corrobora diferencias estadísticamente significativas, entre las mismas.
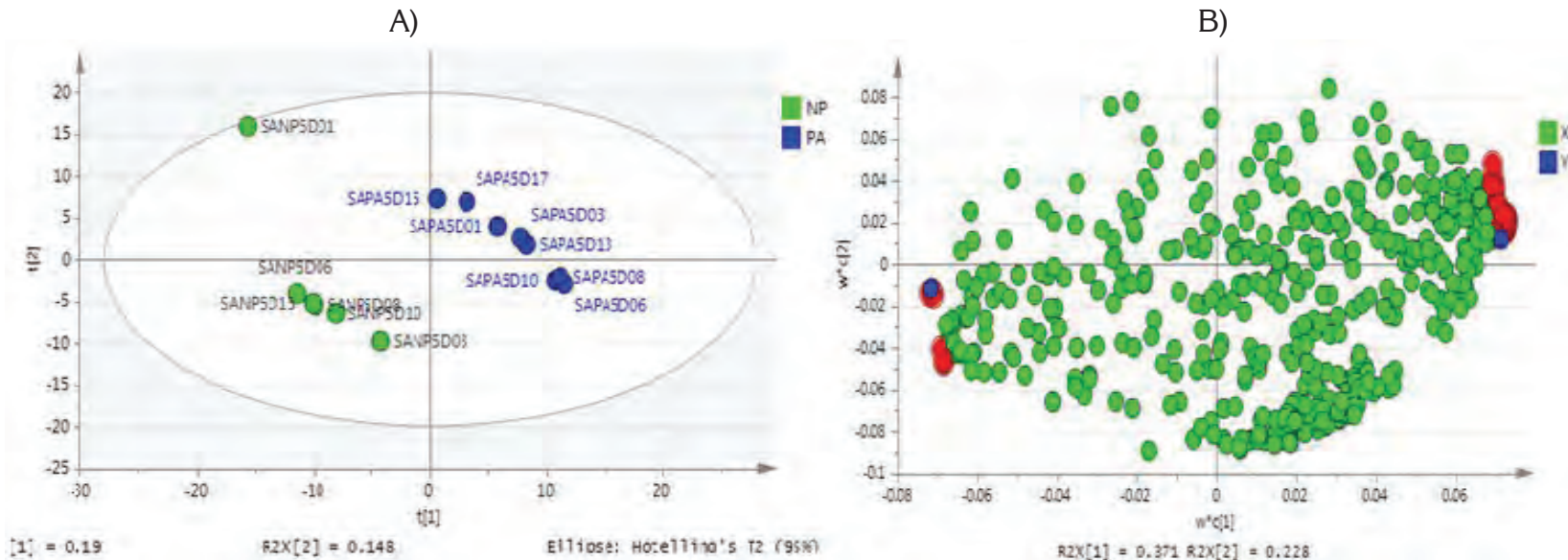

A) Representación bidimensional de la distribución de muestras formuladas con sacarosa, con y sin tratamiento, arrojado por el modelo PCA estimado y B) Representación gráfica de los iones discriminantes (VIP) en el espacio, identificados por el software SIMCA, durante la comparación de muestras de jugo de mora microfiltrado formulado con sacarosa, con y sin tratamiento.

Figura 2. Representación de la distribución de muestras formuladas con sacarosa con y sin tratamiento.

Del mismo modo, el software permitió obtener el listado de iones discriminantes que, para esta comparación, está integrado por 33 iones aportados, inicialmente, el software MZmine, que alcanzaron valores VIP superiores a 1,5. La representación gráfica de los mismos es presentada en la figura $2 \mathrm{~B}$.
COMPARACIÓN C: Cotejo efectuado entre muestras de jugo de mora microfiltrado formulado con estevia y sacarosa sometidos o no a pasteurización: Una vez realizado el análisis global de las intensidades de los diferentes iones presentes en las muestras (tanto formuladas con estevia como con sacarosa, pasteurizadas y no), por medio del soft- 
ware SIMCA, tal y como fue mencionado anteriormente, fue posible visualizar la tendencia de los iones presentes en las muestras, según el tratamiento. Su distribución en el espacio bidimensional puede ser observado en la figura 3A.
Se puede apreciar notoriamente, cómo las muestras pertenecientes a cada categoría, según el tratamiento, se distribuyen de forma similar, agrupándose entre sí, concentrándose de forma diferente en el espacio, las muestras de jugos
A)

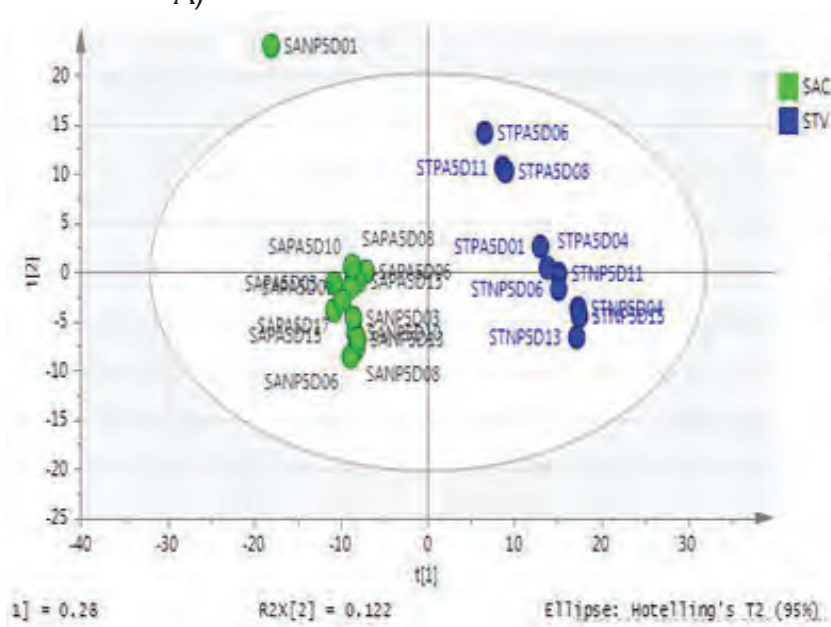

B)

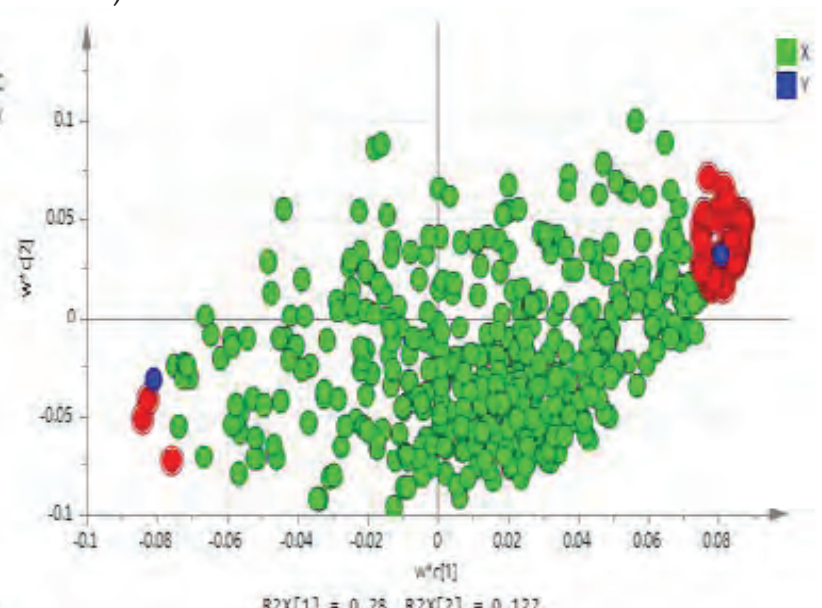

A) Representación bidimensional de la distribución de muestras formuladas con estevia y sacarosa, con y sin tratamiento, arrojado por el modelo. B) Representación gráfica de los iones discriminantes (VIP) en el espacio, identificados por el software SIMCA, durante la comparación de muestras de jugo de mora microfiltrado formulado con estevia y sacarosa, con y sin tratamiento.

Figura 3. Representación de la distribución de las muestras formuladas con estevia y sacarosa, con y sin tratamiento, arrojado por el modelo.

pasteurizados (resaltados en la figura 3A color verde) de los no pasteurizados (azul). Esta variación entre las muestras, según el tratamiento, fue corroborado por el análisis de varianza efectuado, al alcanzar un p valor inferior muy cercano a $0,(3,48 \mathrm{E}-11)$ lo que corrobora diferencias estadísticamente significativas, entre las mismas, con un nivel de confianza de $99,99 \%$, lo que permite afirmar que el tratamiento para las formulaciones estudiadas genera una variación más marcada de los iones presentes, conducta esperada, dada las diferencias existentes en la composición de las muestras, especialmente, gracias a los componentes aportados por la estevia. En este orden de ideas, el listado de iones discriminantes estuvo conformado por 33 iones. La representación gráfica de su ubicación en el espacio puede ser apreciada en la figura 3B.

Investigaciones similares, llevadas a cabo por Malveira (2010), De Paepe et al. (2014), Vervoort et al. (2012) y Toydemir et al. (2013), en las que se emplearon estudios metabolómicos en muestras zumo de melón, jugo de manzana no clarificado, jugo de naranja y jugo de guinda (Prunus cerasus $\mathrm{L}$.), respectivamente, permitieron evaluar, igualmente, como tecnología de procesamiento, el tratamiento térmico. Malveira (2010) fijó, como variables en estudio, el tiempo $(0,16-31 \mathrm{~min})$ y la temperatura $\left(66-94^{\circ} \mathrm{C}\right)$, obteniendo, igualmente, diferencias estadísticamente significativas, entre los metabolitos presentes, en las diferentes muestras. Entre los metabolitos con distribución diferente y, por tanto, ejercieron un efecto significativo en la formación o la degradación del zumo a causa de la pasteurización, se destacan: 4 (1-metilciclohexanol) y 20 (glicina). Por su parte, De Paepe et al. (2014) establecieron, como condiciones del tratamiento, 7.200 segundos en un rango de temperatura de 80 a $145^{\circ} \mathrm{C}$ y evaluaron con mayor especificidad, a través de un enfoque metabolómico no dirigido, basado en espectrometría de masas y cromatografía líquida de alta resolución, los constituyentes fenólicos termolábiles, lo que les permitió descubrir la subclase de algunos de estos compuestos, como la Procyanidin, susceptibles a la degradación térmica.

De igual manera, Toydemir et al. (2013) emplearon, como metodología de análisis, LC-MS, para estudiar los efectos del procesamiento de la fruta de la cereza agria sobre los compuestos polifenólicos. Producto del análisis exhaustivo, los 
autores descubrieron 193 compuestos, en la mencionada fruta, de los cuales, 38 podrían ser identificados y, de éstos, solo siete se vieron afectados por el procesamiento, entre los cuales, cinco corresponden a compuestos fenólicos.

A diferencia de los anteriores, Vervoort et al. (2012) exploraron, como herramienta para comparar el impacto térmico leve del jugo de naranja, GC-MS, revelando diferencias indirectas durante el almacenamiento, que se atribuyen al crecimiento microbiano en el jugo sin tratar y en la inactivación enzimática.

De igual manera, Sugimoto et al. (2012) seleccionaron, como metodología de análisis, un estudio metabolómico, para estudiar el efecto de la pasteurización sobre el sake japonés (vino de arroz). Los resultados de la investigación señalan la disminución de aminoácidos y de polisacáridos, así como el aumento en las concentraciones de péptidos y de glucosa en el sake pasteurizado, determinando, de esta manera, cambios característicos, a causa del tratamiento térmico.

Asimismo, Park et al. (2013) analizaron los componentes volátiles de un producto muy similar, vino de arroz coreano tradicional, no pasteurizado y pasteurizado, durante 30 días de almacenamiento, a través de cromatografía de gases-espectrometría de masas. Un total de 11 compuestos de olor activos, tales como 3-metil-1-butanol (alcohol isoamílico), 2-metil-1-butanol, 2,3-butanodiol, ácido butanoico, ácido 3-metilbutanoico (ácido isovalérico), 2 metilbutanoico, 3 (metiltio) -1-propanol (metionol), 2-feniletanol, decanoato de etilo, dodecanoato de etilo y acetato de tetradecanoat, fueron determinados; la mayoría, se encontraron en mayor proporción en las muestras no pasteurizadas, en comparación con las no pasteurizadas, durante el período de almacenamiento.

Por último, el análisis realizado confirmó la variación de la composición del jugo de mora microfiltrado endulzado, tanto con estevia como con sacarosa, al ser sometidos a una etapa de pasteurización $\left(90^{\circ} \mathrm{C}\right)$. La información cuantitativa y cualitativa obtenida, en relación con el comportamiento del jugo de mora endulzado con estevia, sometido a tratamiento térmico, puede ser utilizado para monitorizar y controlar el procesamiento térmico del producto.

Conflictos de intereses: El manuscrito fue preparado y revisado con la participación de todos los autores, quienes declaramos que no existe conflicto de intereses que ponga en riesgo la validez de los resultados presentados.

\section{BIBLIOGRAFÍA}

1. CAJKA, T.; RIDDELLOVA, K.; TOMANIOVA, M.; HAJSLOVA, J. 2011. Ambient mass spectrometry employing a DART ion source for metabolomic fingerprinting/profiling: A powerful tool for beer origin recognition. Metabolomics. 7(4):500-508.

2. DE PAEPE, D.; VALKENBORGC, D.; COUDIJZER, K.; NOTEN, B.; SERVAES, K.; DE LOOSE, M.; VOORSPOELS, S.; DIELS, L.; VAN DROOGENBROECK, B. 2014. Thermal degradation of cloudy apple juice phenolic constituents. Food Chem. 162:176-185.

3. GARCÍA, C.; HERNÁNDEZ, L.; PÉREZ, A.; VAILLANT, F. 2014. Diversity of urinary excretion patterns of main ellagitannins' colonic metabolites after ingestion of tropical highland blackberry (Rubus adenotrichus) juice. Food Research International. 55:161-169.

4. GONZÁLEZ, C.; TAPIA, M.; PÉREZ, E.; PALLET, D.; DORNIER, M. 2014. Main properties of steviol glycosides and their potential in the food industry: a review. Fruits. 69:127-141.

5. JOHANNINGSMEIER, S.D.; HARRIS, G.K.; KLEVORN, C.M. 2016. Metabolomic technologies for improving the quality of food: Practice and promise. Ann. Rev. Food Science and Technology. 7:413-438.

6. KHAN, W.; CHESTER, K.; ANJUM, V.; AHMAD, W.; AHMAD S.; NARWARIA, A.; KUMAR, D.; KATIYAR, C. 2017. Chromatographic profiling of Pancharishta at different stages of its development using HPTLC, HPLC, GC-MS and UPLC-MS. Phytochemistry letters. Disponible: on line. https:doi.org/10.1016/j. phytol.2017.04.034.

7. KEERTHI, P.; VANKADARI, M.G.; KALAKOTI, S. 2011. Natural sweeteners: A complete review. J. Pharm. Res. 4:2034-2039.

8. KIM, S.; KIM, J.; YUN, E.J.; KIM, K.H. 2016. Food metabolomics: from farm to human. Current Opinion Biotechnology. 37:16-23.

9. MALVEIRA, J. 2010. Avaliação dos efeitos do tratamento térmico sobre o suco de melão: uso da metabolômica para seleção de marcadores. Documento en línea. Disponible desde Internet en: http://www.google. co.ve/url?sa $=$ t\&rct $=j \& q=\&$ esrc $=s \& f r m=1 \&$ sourc $\mathrm{e}=$ web\&cd $=1$ \&ved $=0$ CBsQFjAA\&url =http\%3A\% 
2F\%2Fwww.alice.cnptia.embrapa.br\%2Fbitstream\% 2Fdoc\%2F880989\%2F1\%2FOT10015.pdfEei=b0E GVMK2J4HxgwSbw4Eo\&usg =AFQjCNFpq96OJG OVdKBiaoRB_ghaTG5dQw\&bvm =bv.74115972,d. eXY (con acceso 10/03/2015).

10. OLIVER, S.G.; WINSON, M.K.; KELL, D.B.; BAGANZ, F. 1998. Systematic functional analysis of the yeast genome. TrendsBiotechnol. 16(10):373-378.

11. PARK, H.J.; LEE, S.M.; SONG, S.H.; KIM, Y.S. 2013. Characterization of volatile components in makgeolli, a traditional Korean rice wine, with or without pasteurization, during storage. Molecules. 18(5):53175325.

12. PLUSKAL, T.; CASTILLO, S.; VILLAR, A.; OREŠIČ, M. 2010. MZmine 2: Modular framework for processing, visualizing, and analyzing mass spectrometry-based molecular profile data. BMC Bioinformatics. 11:395406.

13. PRADA, C. 2004. El futuro del control de procesos. Rev. Iber. Autom. Inform. Ind. 1(1):5-14.

14. PURI, M.; SHARMA, D.; TIWARI, A.K. 2011. Downstream processing of stevioside and its potential applications. Biotechnol. Adv. 29:781-791.

15. RODRÍGUEZ-PÉREZ, C.; QUIRANTES-PINÉ, R.; AMESSIS-OUCHEMOUKH, N.; MADANI, K.; SEGURA-CARRETERO, A.; FERNÁNDEZ-GUTIERREZ, A. 2013. A metabolite-profiling approach allows the identification of new compounds from Pistacia lentiscus leaves. J. Pharmaceutical and Biomedical Analysis, 77:167-174.
16. SHAROBA, A.M.; El DESOUKY, A.I.; MAHMOUD, M.H. 2012. Effect of addition some hydrocolloids and sweeteners on flow behavior and sensory properties of papaya-apricot nectar Blends. J. Food Process. Technol. 3:3-8.

17. SUGIMOTO, M.; KANEKO, M.; ONUMA, H.; SAKAGUCHI, Y.; MORI, M.; ABE, S.; SOGA, T.; TOMITA, M. 2012. Changes in the charged metabolite and sugar profiles of pasteurized and unpasteurized japanese sake with storage. J. Agric. Food Chem. 60(10):2586-2593.

18. TOYDEMIR, G.; CAPANOGLU, E.; GOMEZ ROLDAN, M.; DE VOS, R.C.H.; BOYACIOGLU, D.; HALL, R.D.; BEEKWILDER, J. 2013. Industrial processing effects on phenolic compounds in sour cherry (Prunus cerasus L.) fruit. Food Research International. 53(1):218225.

19. VACLAVIK, L.; SCHREIBER, A.; LACINA, O.; CAJKA, T.; HAJSLOVA, J. 2012. Liquid chromatography-mass spectrometry-based metabolomics for authenticity assessment of fruit juices. Metabolomics 8:793-803.

20. VERVOORT, L.; GRAUWET, T.; KEBEDE, B.; VAN DER PLANCKEN, I.; TIMMERMANS, R.; HENDRICKXA, M.; VAN LOEY, A. 2012. Analytical methods headspace fingerprinting as an untargeted approach to compare novel and traditional processing technologies: A case-study on orange juice pasteurisation. Food Chem. 134:2303-2312.

Recibido: Octubre 22 de 2016

Aceptado: Mayo 29 de 2017

Como citar:

González Torrivilla, C.; Hernández, L.; Vaillant Barka, F. 2017. Análisis de un jugo de mora (Rubus adenotrichos) endulzado con estevia (Stevia rebaudina Bertoni), una aproximación metabolómica. Rev. U.D.C.A. Act. \& Div. Cient. 20(1): 121-129. 